\begin{tabular}{|c|l|}
\hline Title & Topology of plane trigonometric curves and the strangeness of plane curves derived from real pseudo-line arrangements \\
\hline Author(s) & Ishikawa, G. \\
\hline Citation & Hokkaido University Preprint Series in Mathematics, 355, 1-18 \\
\hline Issue Date & 1996-11-1 \\
\hline DOI & 10.14943/83501 \\
\hline Doc URL & http://hdl.handle.net/2115/69105 \\
\hline Type & bulletin (article) \\
\hline File Information & pre355.pdf \\
\hline
\end{tabular}

Instructions for use 
Topology of plane trigonometric curves and the strangeness of plane curves derived from real pseudo-line arrangements

\section{Goo Ishikawa}

Series \#355. November 1996 


\section{HOKKAIDO UNIVERSITY PREPRINT SERIES IN MATHEMATICS}

\#330 Y. Kurokawa, Singularities for projections of contour lines of surfaces onto planes, 24 pages. 1996.

\#331 M.-H. Giga and Y. Giga, Evolving graphs by singular weighted curvature, 94 pages. 1996.

\#332 M. Ohnuma and K. Sato, Singular degenerate parabolic equations with applications to the $p$-laplace diffusion equation, 20 pages. 1996.

\#333 T. Nakazi, The spectra of Toeplitz operators with unimodular symbols, 9 pages. 1996.

\#334 B. Khanedani and T. Suwa, First variation of horomorphic forms and some applications, 11 pages. 1996.

\#335 J. Seade and T. Suwa, Residues and topological invariants of singular holomorphic foliations ${ }^{1}, 28$ pages. 1996.

\#336 Y. Giga, M.E. Gurtin and J. Matias, On the dynamics of crystalline motions, 67 pages. 1996.

\#337 I. Tsuda, A new type of self-organization associated with chaotic dynamics in neural networks, 22 pages. 1996.

\#338 F. Hiroshima, A scaling limit of a Hamiltonian of many nonrelativistic particles interacting with a quantized radiation field, 34 pages. 1996.

\#339 N. Tominaga, Analysis of a family of strongly commuting self-adjoint operators with applications to perturbed Dirac operators, 29 pages. 1996.

\#340 A. Inoue, Abel-Tauber theorems for Fourier-Stieltjes coefficients, 17 pages. 1996.

\#341 G. Ishikawa, Topological classification of the tangent developables of space curves, 19 pages. 1996.

\#342 Y. Shimizu, A remark on estimates of bilinear forms of gradients in Hardy space, 8 pages. 1996.

\#343 N. kawazumi and S. Morita, The primary approximation to the cohomology of the moduli space of curves and cocycles for the stable characteristic classes, 11 pages. 1996.

\#344 M.-H. Giga and Y. Giga, A subdifferential interpretation of crystalline motion under nonuniform driving force, 18 pages. 1996.

\#345 A. Douai and H. Terao, The determinant of a hypergeometric period matrix, 20 pages. 1996.

\#346 H. Kubo and K. Kubota, Asymptotic behaviors of radially symmetric solutions of $\square u=|u|^{p}$ for super critical values $p$ in even space dimensions, 66 pages. 1996.

\#347 T. Nakazi and T. Yamamoto, Weighted Norm Inequalities For Some Singular Integral Operators, 17 pages. 1996.

\#348 Y. Ito and I. Nakamura, Hilbert schemes and simple singularities $A_{n}$ and $D_{n}, 22$ pages. 1996

\#349 R. Agemi and K. Yokoyama, The null condition and global existence of solutions to systems of wave equations with different speeds, 42 pages. 1996

\#350 F. Hiroshima, Weak coupling limit with a removal of an ultraviolet cut-off for a Hamiltonian of particles interacting with a massive scalar field, 21 pages. 1996.

\#351 T. Nakazi and Y. Watatani, Invariant subspace theorems for subdiagonal algebras, 22 pages. 1996.

\#352 Y. Nishiura and H. Suzuki, Nonexistence of stable turing patterns with smooth limiting interfacial configurations in higher dimensional spaces, 21 pages. 1996.

\#353 Y.-G. Chen, Y. Giga and K. Sato, On instant extinction for very fast diffusion equations, 9 pages. 1996.

\#354 A. Gyoja and H. Yamashita, Associated variety, Kostant-Sekiguchi correspondence, and locally free $U(\mathrm{n})$-action on Harish-Chandra modules, 25 pages. 1996. 


\title{
Topology of plane trigonometric curves and the strangeness of plane curves
derived from real pseudo-line arrangements
}

\author{
by Goo ISHIKAWA
}

1991 Mathematics Subject Classification: Primary 58C27, 14P25; Secondary 52B30, 51A30, 05B35.

Keywords: trigonometric curve, Hilbert's 16 th problem, pseudo-line arrangement, strangeness, Gauss diagram, Plücker-Klein's formula.

\section{Introduction}

A plane trigonometric curve $f: S^{1} \rightarrow \mathrm{C}$ is a plane curve parametrized by trigonometric polynomials: $f(z)=\sum_{j=-n}^{n} a_{j} z^{j},|z|=1, a_{j} \in \mathrm{C}$. In this note we present an approach to the topological classification of generic trigonometric curves of lower degree and observe its relation to the topology of plane curves derived from a pseudo-line arrangement, namely the union of properly embedded topological lines (strings) on the plane, intersecting transversely at exactly one point to each other.

In particular, we show that a trigonometric curve with the maximal number of self-intersection points among generic trigonometric curves of given degree has no inflection points. It is given the topological classification of generic trigonometric curves of degree 2. Moreover we observe that there exist 14 isotopy types of generic Fourier curves of degree 3 with 10 self-intersection points, and there exists a duality for the strangeness St (in Arnold's sense) on these 14 types of generic plane curves. This fact is generalized to the isotopy types of plane curves derived from simple pseudo-line arrangements 
with fixed odd number of strings such that the sum of St is constant for each dual pair.

In both academic and non-academic lives, we face a huge number of plane curves, for instance, trajectories or contours of some objects. There are, of course, a huge number of mathods to analyze plane curves from various viewpoints. In the mathematical sense, a plane curve has two appearances: as a level curve of a function on the plane, and an image of a parametrization. In former case, Taylor expansion of the function offers effective information about the level curve. In latter case, if the curve is closed, we have, say, a smooth map from the circle to the plane. Then it is a very ordinary method, for the analysis of the plane curve, to use Fourier expansion of the curve, besides of Taylor expansion. Exactly, this is the essential idea of the present note, which treats in particular the topology of plane curves.

Consider a $C^{\infty}$ parametric plane curve $f: S^{1} \longrightarrow \mathbf{R}^{2}$. We identify $S^{1}$ with the unit circle in the complex plane $\mathbf{C}$ and $\mathbf{R}^{2}$ with the whole $\mathbf{C}$. Then, as well known, Fourier expansion of $f$ :

$$
f(z)=\sum_{j=-\infty}^{\infty} a_{j} z^{j},|z|=1, a_{j}=\frac{1}{2 \pi \sqrt{-1}} \int_{|z|=1} f(z) z^{-(j+1)} d z
$$

converges with respect to $C^{\infty}$ topology of $C^{\infty}\left(S^{1}, \mathrm{C}\right.$ ). (For fundamental facts about Fourier series, see [14] for instance. ) If $f$ is generic, namely an immersion with just transverse self-intersections, then $f$ is topologically stable, that is, stable topologically under small perturbations [10]. Remark that, in this case, topologically stability is equivalent to $C^{\infty}$ stability. Therefore the plane curve $f$ has the same isotopy type with a curve defined by some leading terms of Fourier expansion of $f$. Thus any isotopy type of generic plane curves is realized as that of plane curve represented by trigonometric polynomials. Then, for the topological classification problem of generic plane curves, it is natural to classify the isotopy types realized by trigonometric plane curves of a given degree from $1,2,3, \ldots$ and so on.

Let $f: S^{1} \rightarrow \mathrm{C}$ be a plane curve defined by a trigonometric polynomial map $f(z)=\sum_{j=-n}^{n} a_{j} z^{j}$ of degree $\leq n$, where $z=\cos (\theta)+\sqrt{-1} \sin (\theta) \epsilon$ $S^{1} \subset \mathrm{C}$, and $a_{j} \in \mathrm{C},-n \leq j \leq n$. In what follows, we simply call $f$ a trigonometric curve of degree $\leq n$. Thus the space of trigonometric curves of degree $\leq n$ is identified with $\mathrm{C}^{2 n+1}$. Non-generic trigonometric 
curves of degree $\leq n$ form a semi-algebraic set $\Sigma$ in $\mathbf{C}^{2 n+1}$. Then our final goal is to classify generic trigonometric curves $f \in \mathrm{C}^{2 n+1}-\Sigma$ up to isotopy.

The general classification theory of parametric plane curves goes back to Gauss: Gauss words or Gauss diagrams [9][4][8]. Whitney [25] gave essential results on a regular homotopy invariant, that is, an invariant under deformations through immersions: Gauss index or Whitney index, namely, the mapping degree of Gauss map of an immersed plane curve. Scott Carter [4] showed that Gauss diagram is the complete invariant for the plane curves as spherical curves. Arnold [1][2] gave three kinds of basic isotopy invariants $J^{ \pm}$and strangeness St of generic plane curves. These are first order invariants of Vassiliev type [20]. Also there exist many works about plane curves related to the knot theory. See the excellent survey [5]. We concern with this recent theory of topological invariants of parametric plane curves, and intend to apply it to the topology of trigonometric plane curves.

Extending the scope, we remark, for a Fourier curve $f$ of degree $n$, there naturally corresponds a real rational curve $f: \mathbf{R P}^{1} \longrightarrow \mathbf{R P}^{2}$ of degree $2 n$, if we set $\cos (\theta)=\left(1-t^{2}\right) /\left(1+t^{2}\right), \sin (\theta)=2 t /\left(1+t^{2}\right)$, while $t=\tan (\theta / 2)$. This reflects the fact that the algebraic curve $S^{1}=\left\{x^{2}+y^{2}=1\right\} \subset \mathbf{R}^{2} \subset$ $\mathbf{R P}^{2}$ is rational of degree 2. Viro [23] studies the classification problem of real rational curves from Vassiliev's point of view, improving the theory of the strangeness for real rational curves.

On the other hand this classification problem has analogous feature to the 16th problem of Hilbert, in particular, to the first half of it.

The first half of the 16th problem of Hilbert treats, in particular, the classification problem of non-singular real algebraic curves of fixed degree $m$ up to isotopy in the real projective plane $\mathbf{R P}^{2}$. See [21]. Up to present, this problem is solved for $m \leq 7$. For the case $m \leq 5$ the classification is classically known; Gudkov classified for $m=6$ and Viro for $m=7$.

We observe that, in the topological classification problem of real algebraic curves, the strategy is first to find restrictions or estimates of topological invariants and second to find realizations or constructions of given topological types.

Then we are naturally led to the similar topological classification problem of real rational curves and trigonometric curves.

For the theory of topology of real plane algebraic curves, the starting 
point is Harnack's inequality: Harnack showed that the number of connected components of a real algebraic curve of degree $m$ is at most $(1 / 2)(m-1)(m-$ $2)+1$. Then, a non-singular curve of degree $m$ is called, after Petrovskii, an $M$-curve if it has the maximal number of connected components.

Notice that, for the first non-trivial case, namely $m=6$, the concrete topological classification shows clearly certain symmetry or duality. For instance, there exist three isotopy types of $M$-curves of degree 6 as in Fig.1. However, as far as the author know, the full explanation of this duality is not given yet. The purpose of this note is to exhibit similar duality for the first non-trivial case of the topological classification of trigonometric curves or real rational curves.

A real rational curve $f: \mathbf{R P}^{1} \rightarrow \mathbf{R P}^{2}$ is called generic if $f$ is an immersion with only transverse self-intersections. For the basic topological restrictions on the real rational curves, we have the following:

Lemma 1.1 Let $f$. be a generic real rational curve of degree $m$. Then

(1) The number of self-intersection points $\ell \leq(1 / 2)(m-1)(m-2)$.

(2) The number of intersection points with a generic line is at most $m$ and it is congruent to m mod. 2 .

Example 1.2 : Consider the topological classification of generic real rational curves of degree 4. Then, by Lemma 1.1(1), we have the number of self-intersection points $\ell \leq 3$. Moreover using Lemma 1.1(2), we have the list of isotopy types given in Fig.2.

Now we turn to the classification problem of trigonometric curves. Then we have:

Lemma 1.3 Let $f: S^{1} \longrightarrow \mathrm{C}$ be a generic trigonometric curve of degree $n$. Then we have:

(1) The number of self-intersection points $\ell \leq(2 n-1)(n-1)$.

(2) The absolute value $i$ of Gauss index of $f$ is less or equal to $n$ and congruent to $\ell+1$ mod. 2 .

A generic trigonometric curve of degree $n$ is called $M$-curve if $\ell=(2 n-$ $1)(n-1)$. Similarly this definition is applied also to generic real rational curves.

Then the first major result of this note is the following: 
Proposition 1.4 Let $f$ be a generic trigonometric curve of degree $n$. If $f$ is an $M$-curve, then $f$ has no inflection points.

This gives a severe topological restriction on the isotopy types of $M$ curves.

Example 1.5 : There does not exist a trigonometric curve of degree 2 with the topological type as in Fig.3. In fact assume contrarily it does exist. Then it is an $M$-curve, so it does not have any inflection points. However, on the other hand, we see necessarily $i=0$, so the number of inflection points is at least 2. This leads a contradiction.

The list of isotopy types of generic trigonometric curves of degree 2 is the same with the remaining types appeared in the list (Fig. 2) of isotopy types of generic real rational curves of degree 4 .

Next let us turn to classify trigonometric $M$-curves of degree 3 . By Lemma 1.3(2), and Proposition 1.4, we see the following.

Lemma 1.6 Let $f$ be a trigonometric $M$-curve of degree $3(\ell=10)$. Then $f$ has no inflection points and the absolute value of its Gauss index $i=3$.

For the construction of isotopy types, we observe the following result:

Proposition 1.7 There exists a set of 14 isotopy types I of generic trigonometric $M$-curves of degree 3 and an involution $\sigma: I \longrightarrow I$ such that, for any $i \in I, \operatorname{St}(i)+\operatorname{St}(\sigma(i))=2$.

These 14 isotopy types are shown in Fig.4 with the adjacency and the values of Arnold's strangeness St: The duality is represented by the rotation by $\pi$. Notice that this result is obtained by the experiments using ParametricPlot of Mathematica. (It is so easy to handle this and needs little time to draw plane curves, therefore it is rather easy also to find new facts: Compare with an era without such a tool.)

We observe that these 14 isotopy types can be obtained as perturbations of the bouquet or blossom $b_{5}: f=z^{3}-z^{-2}$ with five stalks or petals: Fig. 5. In general we set $b_{2 n-1}: f=z^{n}-z^{-(n-1)}$, which is a non-generic trigonometric curve of degree $n$, and is the bouquet with $(2 n-1)$-stalks or the blossom with $(2 n-1)$-petals. This curve provides the unique topological type of real algebraic curves in $\mathbf{R P}^{2}$ of degree $2 n$ with one real ordinary $(2 n-$ 
1)-multiple point. (This remark was found during a conversation with $T$. Fukui.) Then we get necessarily an $M$-trigonometric curve of degree $n$ if we perturb $b_{2 n-1}$ into an generic trigonometric curve of degree $n$. The existence of the duality, in the case $n=3$, is clear from Fig.4. However we observe that this duality comes from more general connection with the topology of trigonometric curves and the topology of (pseudo-)line arrangements [13] on the real plane $\mathbf{R}^{2}$.

A pseudo-line arrangement $\mathcal{A}$ of $s$ strings in $\mathbf{R}^{2}$ is a proper $C^{\infty}$ immersion $F: \amalg_{s} \mathbf{R} \rightarrow \mathbf{R}^{2}$ of $s$-copies of real lines with the following properties: If we denote by $F_{i}$ the restriction of $F$ to the $i$-th $\mathbf{R}$ and by $L_{1}, \ldots, L_{s}$ the images $F_{1}(\mathbf{R}), \ldots, F_{s}(\mathbf{R})$, then each $F_{i}$ is an embedding, $1 \leq i \leq s$, and $L_{i}$ and $L_{j}, i \neq j$, intersect transversely at exactly one point in $\mathbf{R}^{2}$. A point $p \in \mathbf{R}^{2}$ is called an $m$-multiple point (with respect to $F$ ) if $F^{-1}(p)$ consists of $m$-points in $\amalg_{s} \mathbf{R}$. A pseudo-line arrangement is called simple if there does not exist $m$-multiple points with $m \geq 3$.

Two mappings $F: M \rightarrow N$ and $G: M^{\prime} \rightarrow N^{\prime}$ between topological spaces are called homeomorphic if there exist homeomorphisms $\sigma: M \rightarrow M^{\prime}$ and $\tau: N \rightarrow N^{\prime}$ such that $G \circ \sigma=\tau \circ F$.

$F$ and $G$ are called isotopic if there exist a homeomorphism $\sigma: M \rightarrow M^{\prime}$ and a one-parameter family of homeomorphisms $\tau_{\lambda}: N \rightarrow N^{\prime}(\lambda \in[0,1])$ such that $\tau_{0}=1_{N}$ and that $G \circ \sigma=\tau_{1} \circ F$.

$F$ and $G$ are called strictly isotropic if there exist one-parameter families of homeomorphisms $\sigma_{\lambda}: M \rightarrow M^{\prime}$ and $\tau_{\lambda}: N \rightarrow N^{\prime}(\lambda \in[0,1])$ such that $\sigma_{0}=1_{M}, \tau_{0}=1_{N}$ and that $G \circ \sigma_{1}=\tau_{1} \circ F$.

Any pseudo-line arrangement $F$ of $s$ strings is strictly isotopic to an arrangement $F^{\prime}$ with the following property: there exists a permutation $\rho$ of $\{1, \ldots, s\}$ such that each string $F_{\rho(i)}^{\prime}$ coincides with the straight line $\ell_{i}: \mathbf{R} \rightarrow \mathbf{R}^{2}=\mathbf{C}$ defined by $\ell_{i}(t)=t e^{\sqrt{-1}(i-1) \pi / s}$ outside of a compact subset of $\mathbf{R}$.

We call an arrangement $F^{\prime}$ normarized if it has the above property.

Any pseudo-line arrangement $F$ of an odd number $s=2 n-1$ of strings is isotopic to an arrangement $F^{\prime}$ with the following properties: $F_{1}, \ldots, F_{s}$ coincide with the straight lines

$$
\ell_{1},-\ell_{2}, \ell_{3}, \ldots,-\ell_{2 n-2}, \ell_{2 n-1} \text {. }
$$


See Fig. 6. Here $-\ell_{i}$ is defined by $\left(-\ell_{i}\right)(t)=-\ell_{i}(t)$. In fact we need only a permutation of the components of $\amalg_{s} \mathbf{R}$ and reversing some of $\mathbf{R}$ 's for a normalized representative.

We call a pseudo-line arrangement $F^{\prime}$ of an odd number of strings admissible if it has the above property.

Now we are going to construct two (isotopy types of) closed curves $S^{1} \rightarrow$ $\mathbf{R}^{2}$ from an admissible pseudo-line arrangement $F$ of $(2 n-1)$ strings as follows: Take a circle $C_{r}$ on $\mathbf{R}^{2}$ containing all multiple points of $F$ in the inside and intersecting each string just twice. First start at the point $F_{1}(0)$ and move along $F_{1}$ until we hit $C_{r}$. Then move along the arc counterclockwise till we hit $L_{2}=F_{2}(\mathbf{R})$. Change to $L_{2}$ and draw along $F_{2}$ until we hit $C_{r}$. Then draw along the arc counterclockwise. Continuing this process, we get a (piecewise $C^{\infty}$ ) closed curve $S^{1} \rightarrow \mathbf{R}^{2}$. By smoothing this, we have an immersion $c(F): S^{1} \rightarrow \mathbf{R}^{2}$ of Gauss index $n$. If we move clockwise instead of counterclockwise and in order $F_{1}, F_{s}, F_{s-1}, \ldots$, then we get another plane curve $c^{\prime}(F): S^{1} \rightarrow \mathbf{R}^{2}$ of Gauss index $-n$. We call $c(F)$ and $c^{\prime}(F)$ closures of $F$. See Fig.7. If $F$ is simple, then both $c(F)$ and $c^{\prime}(F)$ are generic and have $(2 n-1)(n-1)$ double points.

If $F$ and $F^{\prime}$ are admissible pseudo-line arrangements and they are isotopic, then the set of isotopy classes $\left\{c(F), c^{\prime}(F)\right\}$ coincides with that of $\left\{c\left(F^{\prime}\right), c^{\prime}\left(F^{\prime}\right)\right\}$. Thus, to an isotopy class $\mathcal{A}$ of pseudo-line arrangement of odd strings, there corresponds a set $\left\{c(\mathcal{A}), c^{\prime}(\mathcal{A})\right\}$ of isotopy classes of closed curves $S^{1} \rightarrow \mathbf{R}^{2}$ as $\left\{c(F), c^{\prime}\left(F^{\prime}\right)\right\}$ for a admissible representative $F$ of $\mathcal{A}$. Then we call $\left\{c(\mathcal{A}), c^{\prime}(\mathcal{A})\right\}$ the closures of $\mathcal{A}$. Remark that there is no preference to choose one of the closures $\left\{c(\mathcal{A}), c^{\prime}(\mathcal{A})\right\}$ of an isotopy class $\mathcal{A}$.

The main result of this note is the following:

Theorem 1.8 Let $\mathcal{A}, \mathcal{A}^{\prime}$ be isotopy types of simple pseudo-line arrangements of $(2 n-1)$ strings and $\left\{c(\mathcal{A}), c^{\prime}(\mathcal{A})\right\},\left\{c\left(\mathcal{A}^{\prime}\right), c^{\prime}\left(\mathcal{A}^{\prime}\right)\right\}$ closures of $\mathcal{A}, \mathcal{A}^{\prime}$ respectively. Then we have

(1) If $c(\mathcal{A})$ or $c^{\prime}(\mathcal{A})$ is isotopic (resp. homeomorphic) to $c\left(\mathcal{A}^{\prime}\right)$ or $c^{\prime}\left(\mathcal{A}^{\prime}\right)$, then $\mathcal{A}$ and $\mathcal{A}^{\prime}$ are isotopic (resp. homeomorphic).

(2) $\operatorname{St}(c(\mathcal{A}))+\operatorname{St}\left(c^{\prime}(\mathcal{A})\right)=n-1$.

(3) $J^{+}(c(\mathcal{A}))=J^{+}\left(c^{\prime}(\mathcal{A})\right)=(n-1)(n-2)$.

(4) $J^{-}(c(\mathcal{A}))=J^{-}\left(c^{\prime}(\mathcal{A})\right)=-(n-1)(n+1)$.

It is natural to define the isotopy invariant strangeness of a pseudo-line 
arrangement $\mathcal{A}$ of $(2 n-1)$ strings as the non-ordered pair

$$
\{k, \ell\}=\left\{\operatorname{St}(c(\mathcal{A})), \operatorname{St}\left(c^{\prime}(\mathcal{A})\right)\right\}
$$

of integers, so that $k+\ell=n-1$.

Let $I$ be the set of isotopy types of generic plane curves $S^{1} \rightarrow \mathrm{C}$ obtained as closures of simple pseudo-line arrangements of $(2 n-1)$ strings. By Theorem 1.8, we have a well-defined involution $\sigma: I \rightarrow I$ by $\sigma(c(\mathcal{A}))=c^{\prime}(\mathcal{A})$, so that, for any $i \in I, S t(i)+S t(\sigma(i))=n-1$.

From (the proof of) Theorem 1.8, we have the following:

Proposition 1.9 If $n$ is even, then $c(\mathcal{A})$ and $c^{\prime}(\mathcal{A})$ are not isotopic (even after the reversing the parameter $\left.S^{1}\right)$. In general, if $c(\mathcal{A})$ and $c^{\prime}(\mathcal{A})$ are isotopic, then, for any admissible representative $F$ of $\mathcal{A}$ and the admissible arrangement $G$ obtained from $F$ by reversing all orientations of $\amalg_{2 n-1} \mathbf{R}$ and rotating by $\pi / s, F$ and $G$ are strictly isotopic.

The essential ingredients of the proof of Theorem 1.8 are Carter's classification theorem of curves by Gauss words [4] and the explicit formulae of $J^{ \pm}$due to Viro [23] and of strangeness due to Shumakovitch [19].

Here is a collection of the known facts in the theory of (pseudo-)line arrangements, related to our problem.

A pseudo-line arrangement corresponds to two embedded graphs on the disk, which are dual to each other in the obvious sense. From Proposition 1.9, we are led to ask on the existence of a self-dual graph.

The line arrangements are of course important examples of pseudo-line arrangements. In particular, by Theorem 1.8, there exists a duality on the set of isotopy types of plane curves $S^{1} \rightarrow \mathbf{R}^{2}$ obtained as a closure from a line arrangement. Remark that this set of isotopy types is contained in the set of isotopy types of curves $S^{1} \rightarrow \mathbf{R}^{2}$ without inflection points.

A pseudo-line arrangement is called stretchable if it is isotopic to a line arrangement [13]. The pseudo-line arrangement shown in Fig.8 is an example of non-stretchable simple pseudo-line arrangement of 9 strings due to Ringel and Grünbaum. As stated in [13], R.J. Canham and E. Halsey showed in 1971 that all simple pseudo-line arrangements of $s$ strings, $s \leq 7$, are stretchable. Grünbaum [13] conjectured that all pseudo-line arrangements of 
$s$ strings, $s \leq 8$, are stretchable. Then Goodman and Pollack [11] showed Grünbaum's conjecture affirmatively, using the method of periodic sequences of permutations.

The numbers $n_{s}$ of homeomorphism types of simple (pseudo-)line arrangements of $s$-strings $(s \leq 7)$ are given, for instance in [18]: $n_{s}=1$, for $s \leq 4$, $n_{5}=6, n_{6}=43, n_{7}=922$.

The number of isotopy types of line arrangements of 5 lines is equal to 7 (Fig.9). The adjacency of these isotopy types is represented by the graph of Fig.10. By Theorem 1.8, we have 14 isotopy types of plane curves with $\ell=10, i=3$. and a duality among these isotopy types, which coincides with the duality found in Proposition 1.7. Moreover we remark that the graph of adjacency (Fig.11) is folded onto the graph of adjacency for line arrangements of 5 lines (Fig.10).

A pseudo-line arrangement $F: \amalg_{s} \mathbf{R} \rightarrow \mathbf{R}^{2}$ is convex (resp. concave) if there exists a closed disk $D_{r} \subset \mathbf{R}^{2}$ containing all multiple points of $F$ such that on $F^{-1}\left(D_{r}\right)$ the determinant $\left|F^{\prime}(t) F^{\prime \prime}(t)\right|>0$ (resp. $<0$.)

If $F$ is convex, then the closure $a(F): S^{\mathbf{1}} \rightarrow \mathbf{R}^{2}$ is a convex curve. If $F$ is concave, then $a^{\prime}(F)$ is concave. The isotopy type of a simple line arrangement are realized by a simple convex (resp. concave) pseudo-line arrangement. This fact causes the duality on a class of isotopy types of plane curves without inflection points.

Related to the existence of non-stretchable pseudo-line arrangements we observe the following result from the singularity theory:

Proposition 1.10 The deformation $\tilde{F}:\left\{\amalg_{9}(\mathbf{R}, 0)\right\} \times \mathbf{R}^{N} \rightarrow \mathbf{R}^{2}$ by all line arrangements of the parametrization $F: \coprod_{9}(\mathbf{R}, 0) \rightarrow \mathbf{R}^{2}, 0$ of straight 9-lines (Fig.12) is not topologically versal.

A deformation of a map-(multi-)germ is topologically versal if it contains all topological types appearing as perturbations of the original germ. See, for instance, [17] [6] [7] for the general study on smooth or topological versality of smooth map-germs.

The proof of Proposition 1.10 is a easy consequence of Puppus' theorem (Fig.13). By an infinitely flat perturbation of $\tilde{F}$, we get a non-stretchable pseudo-line arrangement as a perturbation of $F$. Originally this example was found by Levy [13]. 
Based on Goodman-Pollack's theorem [11], we conjecture that the deformation by all line arrangements of straight $s$-lines is topologically versal for $s \leq 8$.

Two important questions remain open to make closer the connection between the topology of trigonometric curves and the topology of pseudo-line arrangements: (1) Set $f=z^{n}-z^{-(n-1)}: S^{1} \rightarrow$ C. Then is the unfolding $F: S^{1} \times \mathbf{C}^{2 n+1} \rightarrow \mathbf{C} \times \mathbf{C}^{2 n+1}$ of $f$ defined by $F(z, a)=f(z)+\sum_{j=-n}^{n} a_{j} z^{j}, a=$ $\left(a_{j}\right)_{j=-n}^{n}$, topologically versal? (2) Is any closure of a pseudo-line arrangement of $(2 n-1)$-strings realizable as a perturbation of $f=z^{n}-z^{-(n-1)}$ in $C^{\infty}\left(S^{1}, \mathbf{C}\right) ?$

We remark that the example (Fig.8) of simple non-stretchable pseudoline arrangement is realized by a convex pseudo-line arrangement. If the above question (2) is affirmative, then we see, as a corollary, that any isotopy type of simple pseudo-line arrangement of odd number of strings is realized by a convex (or concave) pseudo-line arrangement.

We will treat these fundamental questions in a forthcoming paper.

For the concrete topological classification of real rational curves of degree 6 in $\mathbf{R P}^{2}$, it seems that few results are known besides trivial observations. Here we just mention that, using Petrovsii-Marin's inequality [16], we see the left picture of Fig.14 is not realized as an isotopy type of real rational curve of degree 6 . However this method can not applied to the right picture of Fig.14. The author does not know whether it is realizable or not.

Recently there appeared an important paper [3] related, at least in title and in principle, to the present paper. In that paper Arnold classifies holomorphic mappings $\mathbf{C P}^{1} \rightarrow \mathbf{C P}^{1}$ with exactly two preimages of some point in the target. Therefore he treats the topology of the complexifications (and the projectification) of real trigonometric polynomial functions $\mathbf{R} \rightarrow \mathbf{R}$, while we are treating the topology of pairs of real trigonometric polynomial functions. See also [12].

In the next section we give proofs of all results, except for the experimental result Proposition 1.7.

Acknowledgement: The author is very grateful to Professors S. Duzhin, 
T. Fukui, S. Izumiya, S. Jimbo, S. Koike, T. Ohmoto, T. Ozawa, C.T.C. Wall and K. Watanabe for helpful information and encouragement. He wish to thank Professor M.A.S. Ruas for her giving him the opportunity to have a talk in the Workshop and for the warm hospitality in Saõ Carlos.

The first draft of this paper appeared in the series of mimeographical notes of RIMS of Kyoto University: "Sûrikaiseki-kenkyûsho Kôkyûroku" 952, (1996).

\section{Proofs of Results}

Proof of Lemma 1.1: (1) follows from the well known genus formula. (2) follows from the theorem of Bezóut.

Proof of Lemma 1.3: (1) follows from Lemma 1.1(1). For (2), we observe the following formula and apply Lemma 1.1(2) to a line through the origin: $d f / d \theta=\sum_{i=-n}^{n} \sqrt{-1} i a_{i} z^{i}$. (The differential defines on $\mathbf{C}^{2 n+1}$ just a diagonal linear action with eigenvalues $-\sqrt{-1} n, \ldots, \sqrt{-1} n$.) Then we have $2 i \leq 2 n$, hence $i \leq n$. The latter congruence follows from Whitney's formula [25].

Proof of Proposition 1.4: Let $C \subset \mathrm{P}^{2}$ be a generic algebraic front, that is, algebraic curve with ordinary double points and ordinary cusps as singularities, of degree $m$. Recall Plücker-Klein's formula [15][22][24]:

$$
\begin{aligned}
k & =m(m-1)-2 d-3 r, \\
m & =k(k-1)-2 t-3 w, \\
w & =3 m(m-2)-6 d-8 r, \\
r & =3 k(k-2)-6 t-8 w .
\end{aligned}
$$

(Here we need the first equality.) And if $C$ is defined over $\mathbf{R}$,

$$
m+w^{\prime}+2 t^{\prime \prime}=k+r^{\prime}+2 d^{\prime \prime} .
$$

Here $k$ is the degree of the dual $C^{\vee}$ in the dual projective plane $\mathbf{P}^{2 *}, d$ is the number of double points, $r$ that of cusps, $t$ double tangents and $w$ inflection points of $C$. The genericity condition demands that all appearing numbers 
are finite. We denote by $w^{\prime}$ (resp. $r^{\prime}$ ) the number of real inflection points (resp. real cusp points). For real curves, remark that there are two kinds of real double points (resp. real double tangents): $t^{\prime \prime}$ designates the number of isolated double tangents, that is, real tangent lines to imaginary points of $C$, and $d^{\prime \prime}$ the number of isolated double points of $C$.

Remark that, if there is a trigonometric $M$-curve with an inflection point, then there is a trigonometric $M$-curve with the same degree satisfying the genericity conditions of Plücker-Klein formula for the image in $\mathbf{P}^{2}$.

Now, for a generic trigonometric curve of degree $n$, consider the corresponding real rational curve and its image in $\mathbf{P}^{2}$. Then $m=2 n, d=\ell=$ $(2 n-1)(n-1), r=0$. So $k=2(2 n-1)$. Since $r^{\prime}=0, d^{\prime \prime}=0$, we have $2 n+w^{\prime}+2 t^{\prime \prime}=2(2 n-1)$, therefore $w^{\prime}+2 t^{\prime \prime}=2 n-2$. But, for trigonometric curve of degree $n$, the line of infinite is perturbed to $(n-1)$ real isolated double tangents. Thus $t^{\prime \prime} \geq n-1$. Therefore we see $w^{\prime}=0$. (Using a generalized version [24], we can see this without perturbations. )

Proof of Lemma 1.6: By Lemma 1.3(2), we have $i=1$ or 3. Moreover, by Proposition 1.4, we see there are no inflection points. Therefore, if $i=1$, then $f$ must be a simple closed curve, which contradicts to $\ell \neq 0$. Thus we have $i=3$.

To prove Theorem 1.8 (1), we use Carter's theorem [4] [5]. We need to recall the notion of Gauss diagram of a generic immersion, and introduce that of a pseudo-line arrangement.

Let $N$ be an oriented one-dimensional manifold and $F: N \rightarrow \mathbf{R}^{2}$ a generic immersion. Take, on $N$, the pairs of inverse images of intersection points determined by $F$. Connect each pair by an oriented chord according to Gauss rule: Let $p, q \in N$ with $F(p)=F(q), p \neq q$. Take positively oriented non-zero vectors $u \in T_{p} N, v \in T_{q} N$ respectively. We connect from $p$ to $q$ if two vectors $F_{*}(u), F_{*}(v)$ form a negative basis of $T_{F(p)} \mathbf{R}^{2}$ with respect to the orientation of $\mathbf{R}^{2}$.

The obtained diagram consisting of $N$ and oriented chords is called Gauss diagram of $F$ and denoted by $D(F)$. For instance Fig.15 indicates Gauss diagrams of curves No.14 and No.1 in Fig.4. In Gauss diagram $D(F)$, we call a compact component of $N$ a circle, and a non-compact component a string.

For an admissible representative $F$ of a pseudo-line arrangement $\mathcal{A}$ of 
$(2 n-1)$-strings, we define Gauss diagram $D(F)$ as Gauss diagram of $F$ as a generic immersion, endowed with the cyclic order of $(2 n-1)$-strings. Then, by Carter's construction in [4], we have

Lemma 2.1 Gauss diagram $D(F)$ (resp. $D(F)$ up to total reversing of cyclic order of strings) determines the isotopy type (resp. homeomorphism type) of $\mathcal{A}$.

Proof of Lemma 2.1: Remark that Carter's method works in our non-compact situation or relative situation as well.

Add to $D(F)$ an extra oriented circle $\partial D$ according to the given cyclic order of strings, such that $\partial D$ intersects to each strings at exactly two points sufficiently far to each other and $\partial D$ does not intersect to any chords. After deleting two ends of each strings, and attaching 2-cells as in [4], we have a 2-complex $D$ and an orientation preserving homeomorphism from $D$ to the unit closed disk in $\mathbf{R}^{2}$. The attach the trivial ends. Thus we obtain a pseudo-line arrangement that recovers the isotopy type of $\mathcal{A}$.

To prove Theorem 1.8 (2), (3), (4), we recall the explicit formulae of $J^{ \pm}$ by Viro [23] and of strangeness by Shumakovitch [19]. See [5].

Let $C$ be an oriented generic immersed closed curve in the plane. Assign an integer $\operatorname{ind}_{C}(Y)$ to each component $Y$ of the complement $\mathbf{R}^{2}-C$ as follows: Assign 0 to the outer component. If we move from $Y$ to neighboring $Y^{\prime}$, while $C$ moves from left to right (resp. from right to left), then ind ${ }_{C}\left(Y^{\prime}\right)=$ $\operatorname{ind}_{C}(Y)+1\left(\right.$ resp. $\left.\operatorname{ind}_{C}\left(Y^{\prime}\right)=\operatorname{ind}_{C}(Y)-1\right)$. Further, for the smoothing $\tilde{C}$ of $C$ with respect to the orientation of $C$, we can assign $\operatorname{ind}_{\tilde{C}}(X)$ to each component of $\mathbf{R}^{2}-\tilde{C}$ compatibly. The Viro's formula says

$$
J^{+}(C)=1+\ell-\sum_{X} \operatorname{ind}_{\tilde{C}}^{2}(X) \chi(X), \quad J^{-}(C)=1-\sum_{X} \operatorname{ind}_{\tilde{C}}^{2}(X) \chi(X),
$$

where $\ell$ is the Gauss index of $C$ as before, and $\chi(X)$ denotes the Euler characteristic of $X$. For a vertex $v$ of $C$, denote by ind $\operatorname{ind}_{C}(v)$ the arithmetic mean of neighboring four $\operatorname{ind}_{C}(Y)$. Take the starting point on an outer edge on $C$. If $C$ passes through $v$ at first time, while another branch of $C$ passes through $v$ from left to right, we set $s(v)=1$; otherwise $s(v)=-1$. Then Shumakovitch's formula says

$$
\operatorname{St}(C)=\sum_{v} s(v) \operatorname{ind}_{C}(v) .
$$


We use also the following non-trivial result:

Lemma 2.2 Two simple admissible pseudo-line arrangements of $(2 n-1)$ strings are connected by a one-parameter family of admissible pseudo-line arrangements with only bifurcations of triple points (without tangent points).

In the case of line arrangements, Ringel [18] showed the corresponding result to Lemma 2.2 .

Proof of Lemma 2.2: First we connect a pseudo-line arrangement $F$ to $F^{\prime}$ such that on the left side of $L_{1}^{\prime}=F_{1}^{\prime}(\mathbf{R})$ there are no intersection points of $F^{\prime}$. To do this we remark that, if there is an intersection point of $F$ on the left side of $L_{1}=F_{1}(\mathbf{R})$, then there is at least one triangle having a side on $L_{1}$ (see Page $50(* *)(i i)$ of [13]). Then we deform $L_{1}$, fixing other strings, through pseudo-line arrangement, along this triangle, so that the number of intersection points on the left side of the string decreases by one.

Next we connect $F^{\prime}$, fixing $F_{1}^{\prime}(\mathbf{R})$, to $F^{\prime \prime}$ having no intersection points on the right side of $F_{2}^{\prime \prime}(\mathbf{R})$ except for the intersection points on $F_{1}^{\prime \prime}(\mathbf{R})=F_{1}^{\prime}(\mathbf{R})$. Iterating this procedure, we connect $F$ to the "standard" arrangement, consisting of tangent lines to a semi-circle with appropriate orientations.

Proof of Theorem 1.8: Let $F$ be an admissible representative of $\mathcal{A}$. First we remark that, We are going to show that the Gauss diagram $D(c(F))$ of the closure of $F$ determines the Gauss diagram $D(F)$ endowed with cyclic order of strings.

In general, the circle of Gauss diagram of a closure of a pseudo-line arrangement of $(2 n-1)$-strings is naturally divided into $(2 n-1)$-arcs. On each arc, there are $(2 n-2)$ intersection points, that is, end points of chords. Moreover each pair of arcs has exactly one pair of intersection points, which is connected by a chord.

If we fix arbitrary division point, which is not an intersection point, then we get a possibly different decomposition of the circle into $(2 n-1)$-arcs, each of which possesses $(2 n-2)$ intersection points on it. We are going to show that the decomposition is uniquely determined, if we impose the condition that each pair of arcs is connected by exactly one chord. Remark that the condition implies that any pair of intersection points on a same arc is not connected by any chord. 
Assume that a division point $P$ (for possible another pseudo-line arrangement) lies on the string $L_{1}$ of $F$ and there are $r, 0<r<2 n-2$, intersection points next to $P$ on $L_{1}$. Consider the end point $Q$ of $L_{1}$, the start point $R$ of $L_{2}$ and the next division point $S$ on $L_{2}$. See Fig.16. Let $T$ be the (image of) intersection point of $L_{1}$ and $L_{2}$.

Assume first that $T$ lies before $P$ on $L_{1}$. Then we have the cycle $T P Q R T$. Consider the $r$ strings intersecting to $L_{1}$ along $P Q$. They must intersect to $L_{2}$ along $R T$. Since $P S$ is a string for another arrangement, $T$ must come after $S$. Besides of $L_{1}$, the $r$ strings intersect to $L_{2}$ on $T S$. Moreover there are $(2 n-2-r)$ intersection points on $R S$, among $(2 n-2)$ intersection points on $P Q R S$. Thus we have $(2 n-2-r)+(r+1)=2 n-1$ intersection points on $L_{2}$. This leads to a contradiction.

So $T$ must lie after $P$ on $L_{1}$. Then $S$ must lie before $T$ on $L_{2}$. However, then, considering $(2 n-2-r)$ strings through $R S$, we have again a contradiction.

(1): If $c(F)$ and $c\left(F^{\prime}\right)$ are isotopic (resp. homeomorphic), then there exists an orientation preserving (resp. not necessarily orientation preserving) isomorphisms of diagrams $D(c(F))$ and $D\left(c\left(F^{\prime}\right)\right)$. Then the isomorphism induces that of $D(F)$ and $D\left(F^{\prime}\right)$ compatible with cyclic order of strings (resp. up to total reversing of cyclic order of strings). By Lemma $2.1, F$ and $F^{\prime}$ are isotopic (resp. homeomorphic).

$(2),(3) \&(4)$ : Under the passage of a triple point, the value $\operatorname{St}(c(F))+$ $\operatorname{St}\left(c^{\prime}(F)\right)$ (resp. $\left.J^{ \pm}(c(F))\right)$ remains constant: If $\operatorname{St}(c(F))$ varies by \pm 1 then $\operatorname{St}\left(c^{\prime}(F)\right)$ varies by $\mp 1$. By concrete computations based on the results of Viro [23] and Shumakovitch [19], the constant is equal to $n-1$ (resp. $(n-1)(n-2)$, $-(n-1)(n+1))$. For instance, for a line arrangement $F$ derived from an equilateral $(2 n-1)$-gon, we have

$$
\left\{\operatorname{St}(c(F)), \operatorname{St}\left(c^{\prime}(F)\right)\right\}=\left\{\sum_{j=1}^{n-1} j(2 n+1-4 j), \sum_{j=1}^{n-1}(1-j)(2 n+1-4 j)\right\},
$$

therefore $\operatorname{St}(c(F))+\operatorname{St}^{\prime}\left(c\left(F^{\prime}\right)\right)=\sum_{j=1}^{n-1}(2 n+1-4 j)=n-1$.

Proof of Proposition 1.10: In the deformation $\tilde{F}$ of $F$ (Fig. 12) by all line arrangements, arbitrarily near $F$, we have line arrangements of nine lines appearing in Puppus' theorem as in Fig.13. By an infinitely flat perturbations of $\tilde{F}$, we deform the ninth line slightly to make a non-stretchable arrange- 
ment, for each line arrangement. This shows $\tilde{F}$ is never topologically versal.

\section{References}

[1] V.I. Arnold, Topological Invariants of Plane Curves and Caustics, University Lecture Series 5, Amer. Math. Soc., 1994.

[2] V.I. Arnold, Plane curves, their invariants, perestroika and classifications, Advances in Soviet Math. 21, Singularities and Bifurcations, Amer. Màth. Soc., (1994), pp. 33-91.

[3] V.I. Arnold, Topological classification of complex trigonometric polynomials and combinatorics of graphs with equal number of vertices and edges, Funct. Anal. Appl., 30 (1996), 1-18.

[4] J.S. Carter, Classifying immersed curves, Proc. Amer. Math. Soc., 111-1 (1991), 281-287.

[5] S. Chmutov, S. Duzhin, Explicit formulas for Arnold's generic curve invariants, Preprint.

[6] J.N. Damon, Topological triviality and versality for subgroups of $A$ and $K$, Memoires of Amer. Math. Soc. 75-389 (1988).

[7] J.N. Damon, Topological triviality and versality for subgroups of $A$ and $K$ : II. Sufficient conditions and applications, Nonlinearity 5 (1992), 373-412.

[8] G. Gairns, D.M. Elton, The planarity problem for signed Gauss words, J. Knot T. and Ramif., 2-4 (1993), 359-367.

[9] C.F. Gauss, Werke VIII, pp. 271-286.

[10] M. Golubitsky, V. Guillemin, Stable Mappings and Their Singularities, Springer-Verlag, (1973).

[11] J.E. Goodman, R. Pollack, Proof of Grünbaum's conjecture on the stretchability of certain arrangements of pseudolines, J. Comb. Theory, Ser. A 29 (1980), 385-390.

[12] V. Goryunov, C. Baines, Möbius and odd real trigonometric $M$-functions, Preprint, Univ. of Liverpool, (1996). 
[13] B. Grünbaum, Arrangements and Spreads, Regional Conf. in Math., 10, Amer. Math. Soc., (1972).

[14] G.H. Hardy, W.W. Rogosinski, Fourier Series, 3rd. ed., Cambridge Tracts in Math. and Math. Physics, 38, Cambridge Univ. Press, (1956).

[15] F. Klein, Eine neue Relation zwischen den Singularitäten einer algebraischen Kurve, Math. Ann. 10 (1876), 199-210 = ges. Abh. Bd. 2, pp. 78-88.

[16] A. Marin, Quelques remarques sur les courbes algebriques planes reelles, in Seminaire sur la geometrie algebrique reelle, ed. by J.-J. Risler Publications Mathematiques de 1'Universéte Paris VII (1980), pp. 51-68.

[17] J. Martinet, Deploiements versels des applications différentiables et classification des applications stables, in Singularit' es d'Applications Différentiables, ed. O. Barlet, F. Ronga, Lecture Notes in Math., 535, (1976), pp. 1-44.

[18] G. Ringel, Über Geraden in allgemeiner Lage, Elem. Math. 12 (1957), 75-82.

[19] A. Shumakovitch, Explicit formulas for strangeness of plane curves, Preprint, Uppsala Univ., 1995:15.

[20] V.A. Vassiliev, Complements of Discriminants of Smooth Maps: Topology and Applications, Revised ed., Transl. Math. Monographs 98, Amer. Math. Soc., (1994).

[21] O. Ya. Viro, Progress in the topology of real algebraic varieties over last six years, Russian Math. Surveys, 41-3 (1986), 55-82.

[22] O. Ya. Viro, Some integral calculus based on Euler characteristic, Lecture Notes in Math. 1346 Springer (1988), pp. 127-138.

[23] O. Ya. Viro, First degree invariants of generic curves on surfaces, Preprint, Uppsala Univ., 1994:21.

[24] C.T.C. Wall, Duality of real projective plane curves: Klein's equation, Topology, 35-2 (1996), 355-362.

[25] H. Whitney, On regular closed curves in the plane, Compositio Math., 4 (1937), 276-284. 
Goo ISHIKAWA

Department of Mathematics, Hokkaido University, Sapporo 060, JAPAN

e-mail: ishikawa@math.hokudai.ac.jp 
Fig.1:

$$
\begin{aligned}
& \text { (0) } \underbrace{0 \ldots 0}_{9} \underbrace{0 \ldots 9}_{9} 0 \\
& \text { Q-9) ?-9 }
\end{aligned}
$$

Fig.2:
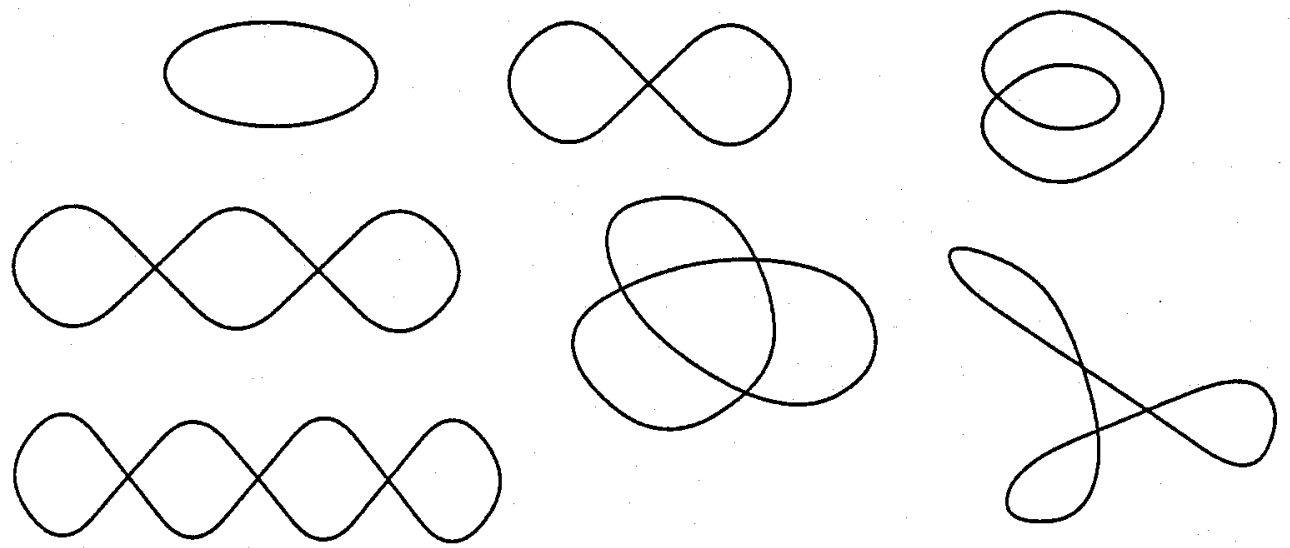

Fig.3:
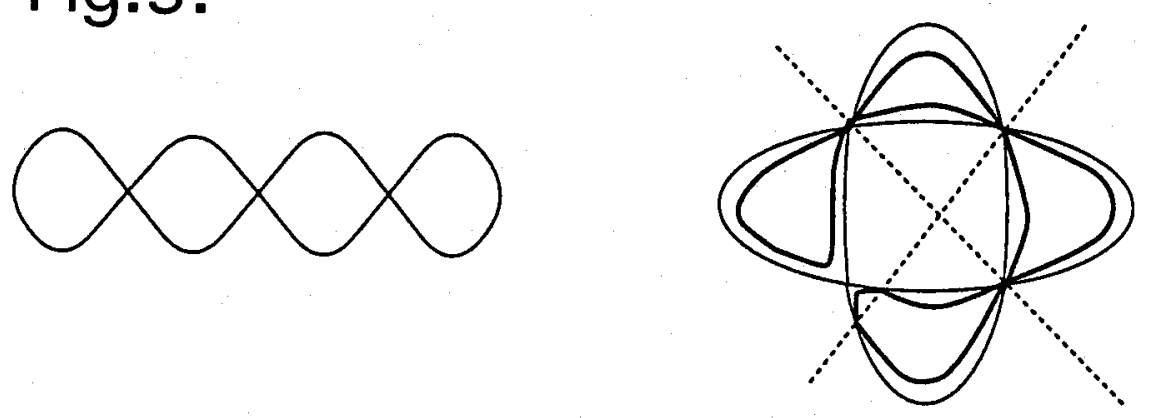
Fig.4:

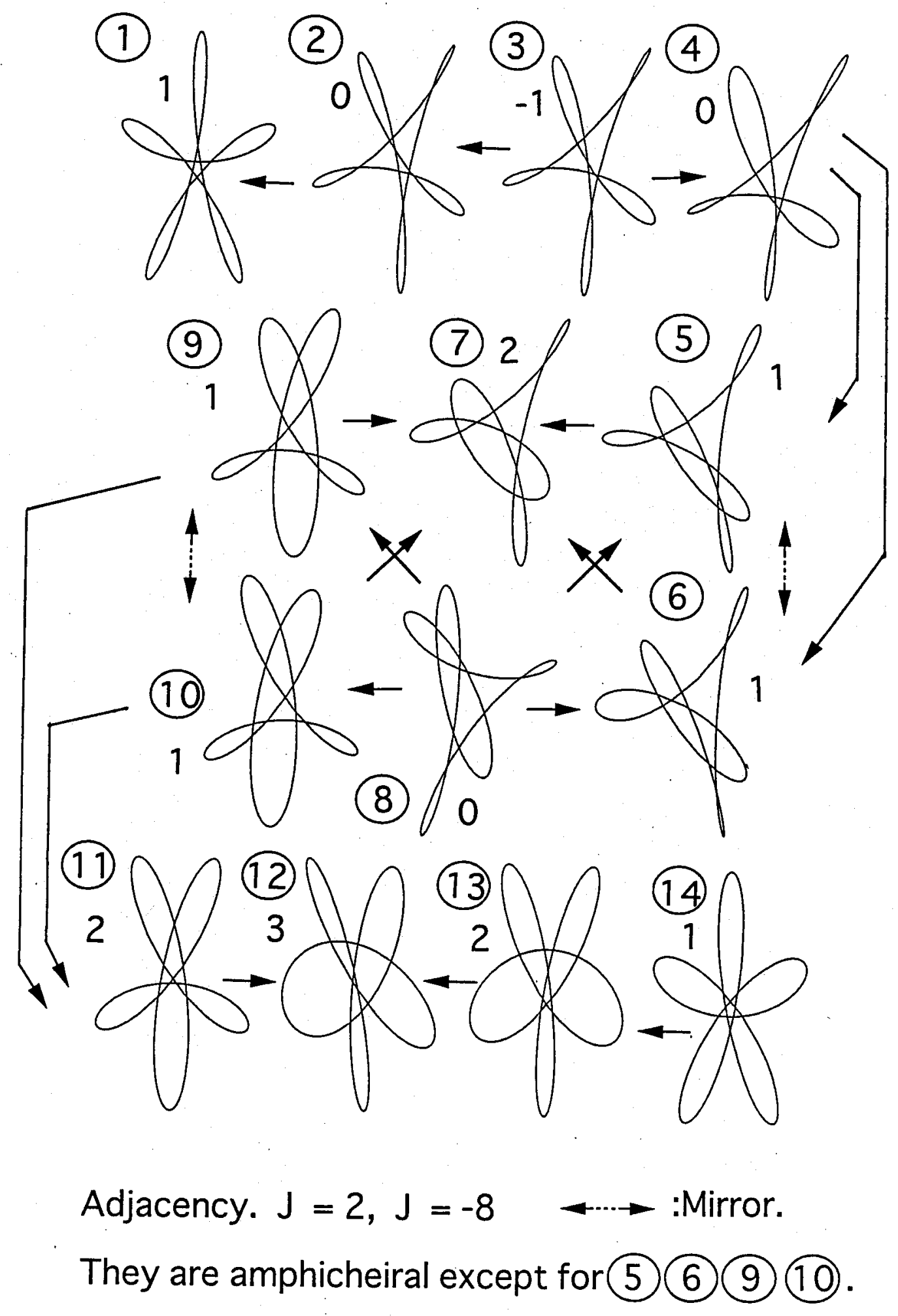


Fig.5:

Fig.6:
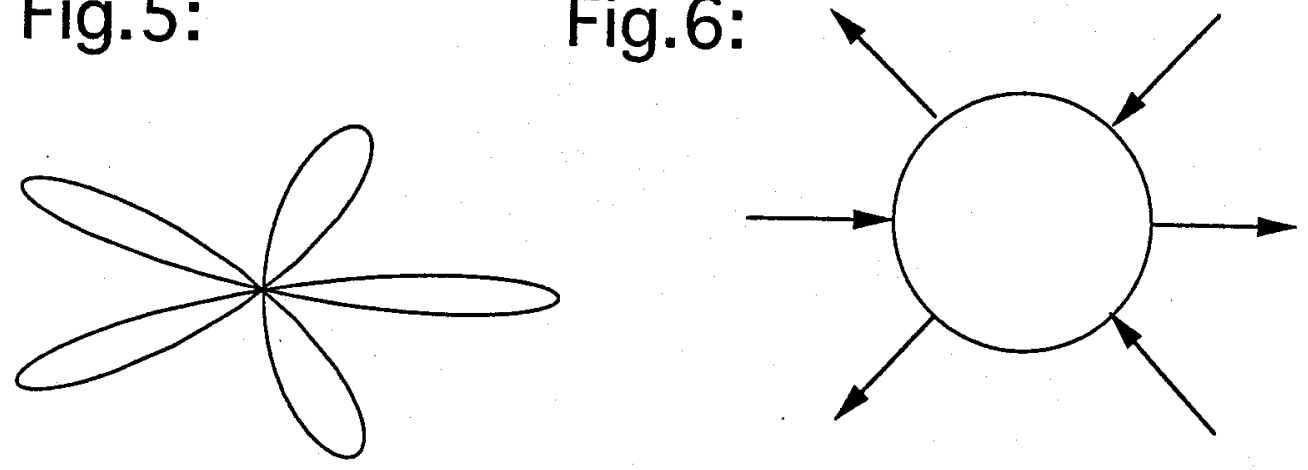

Fig.7: Closures
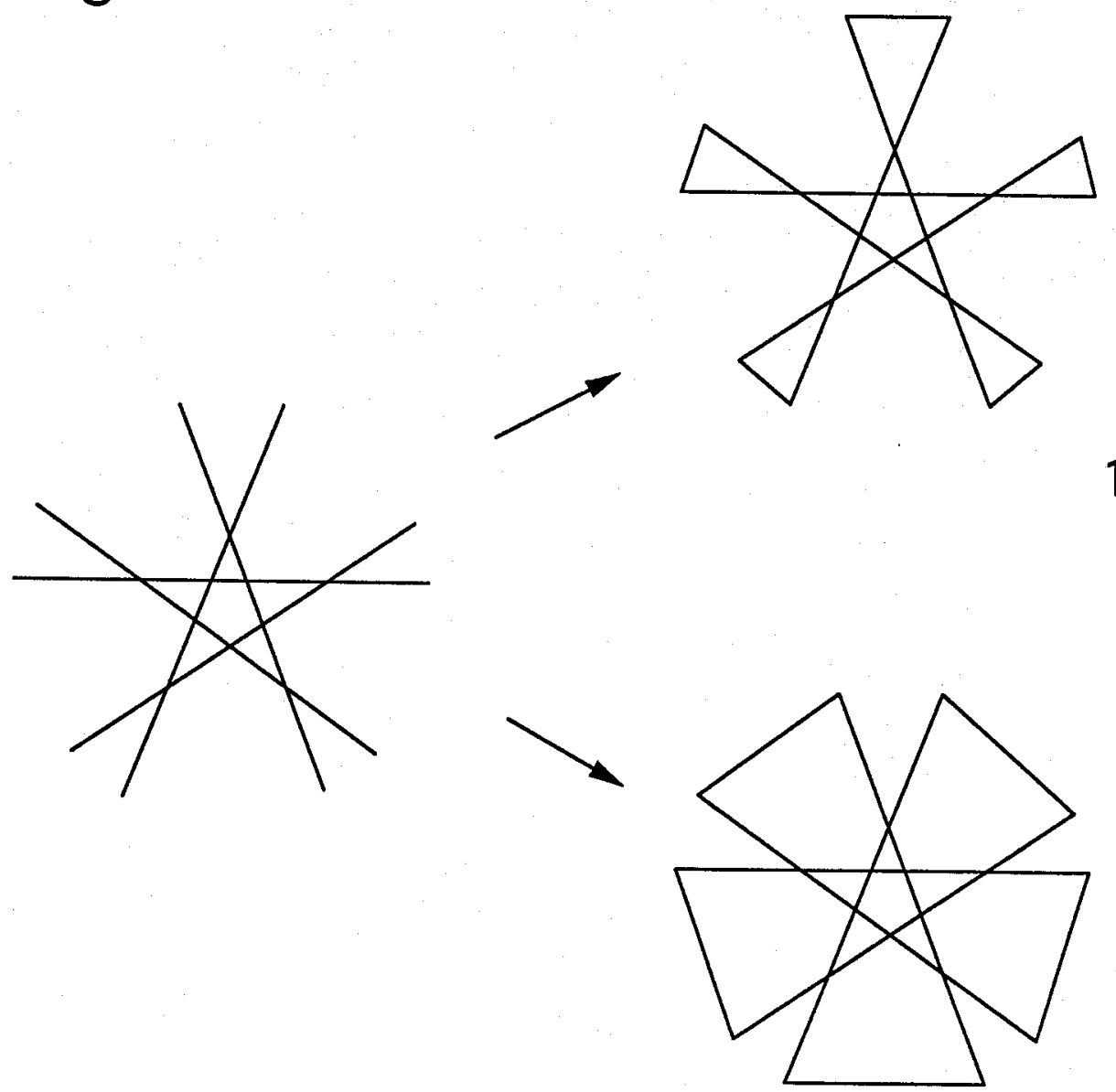

14 
Fig.8:

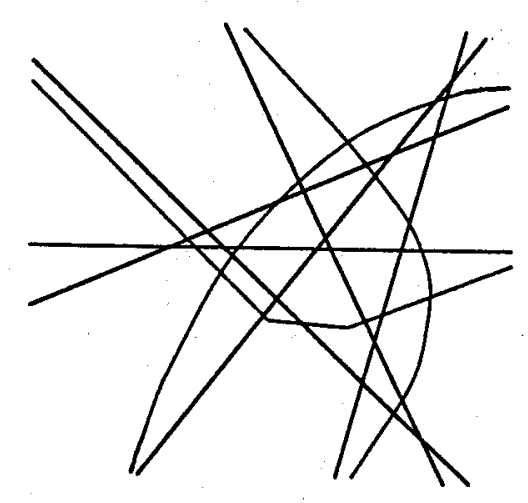

Fig.9:

(Pseudo-)line arrangements with $s=5$.
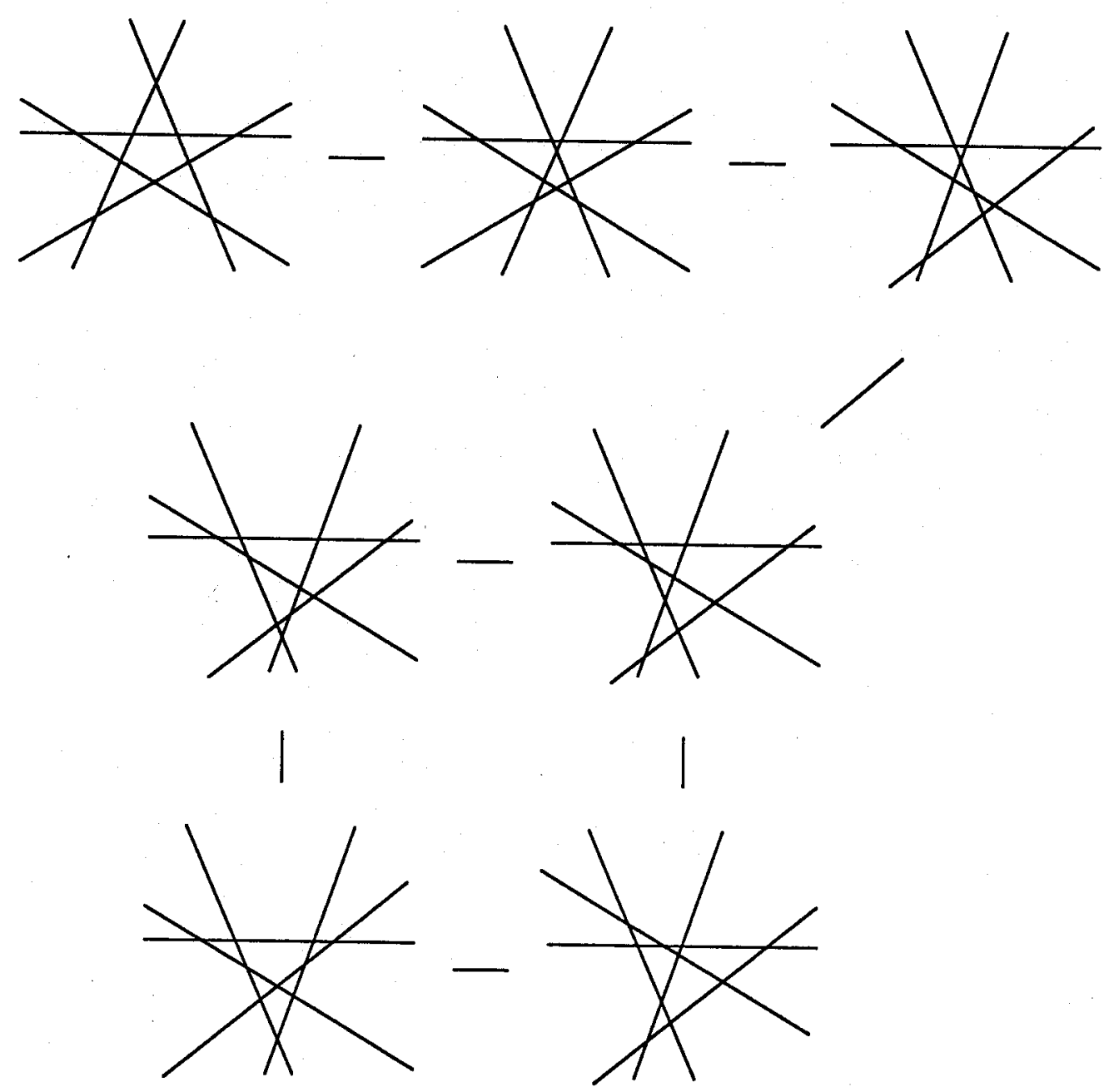
Fig. 10:

Fig.11:
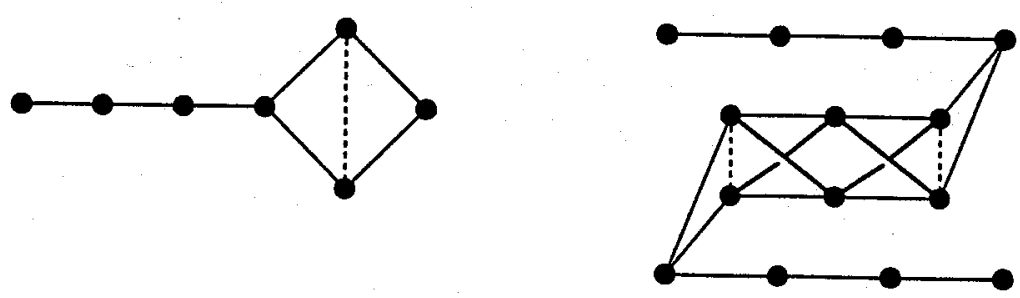

Fig.12:

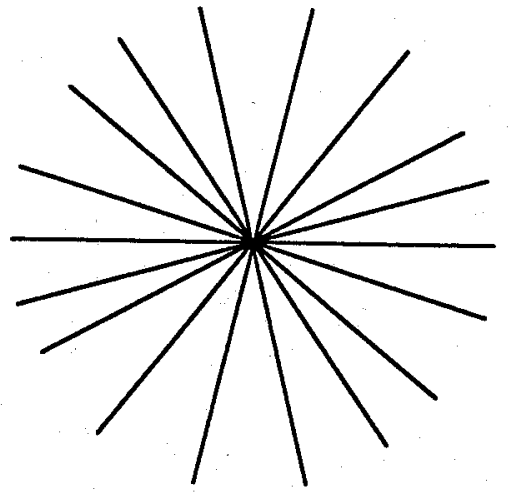

Fig. 13:

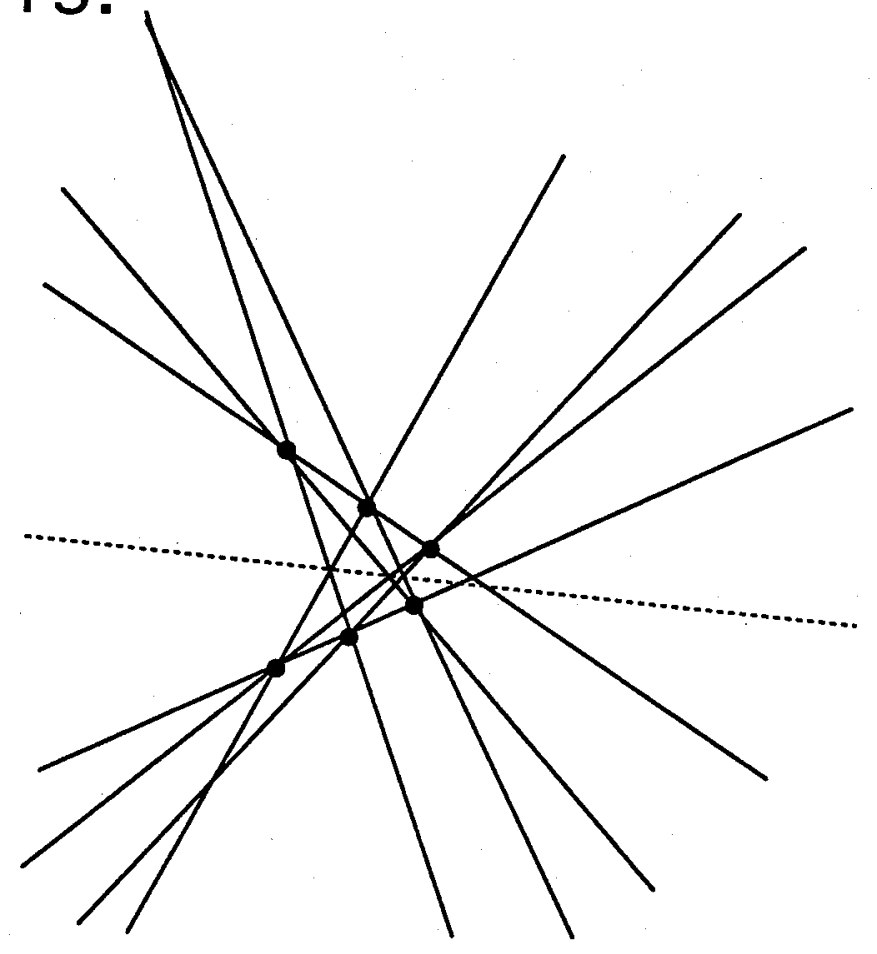


Fig. 14:

$\infty 000000000000010$

Fig.15:
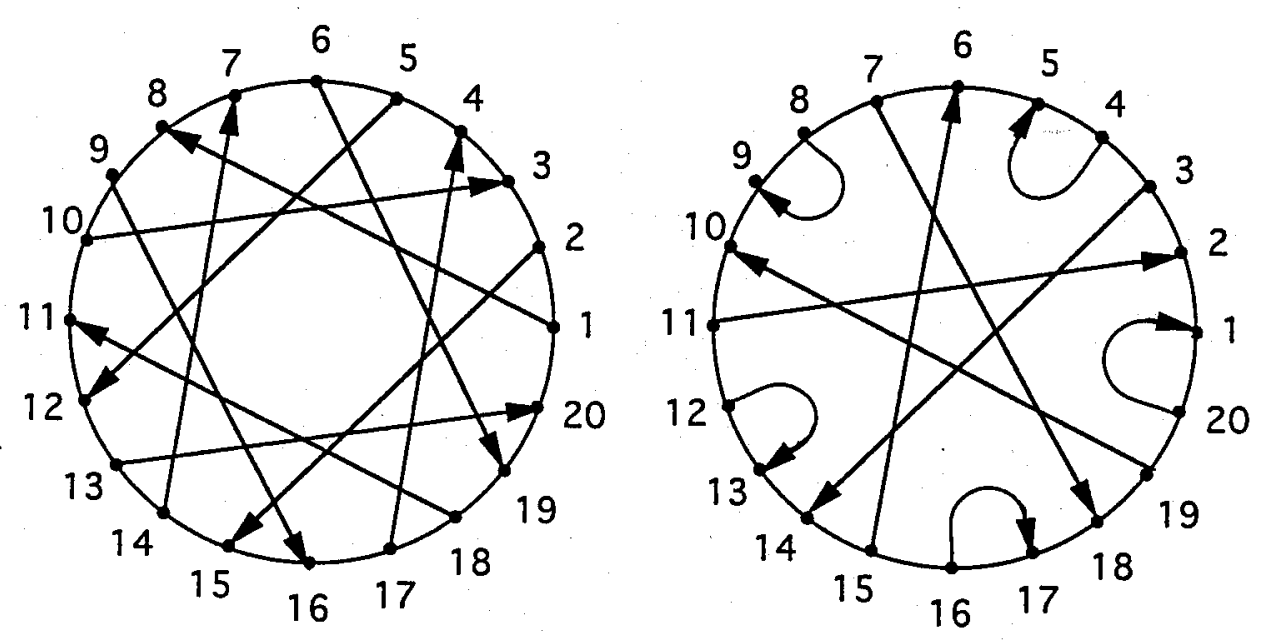

Fig.16:

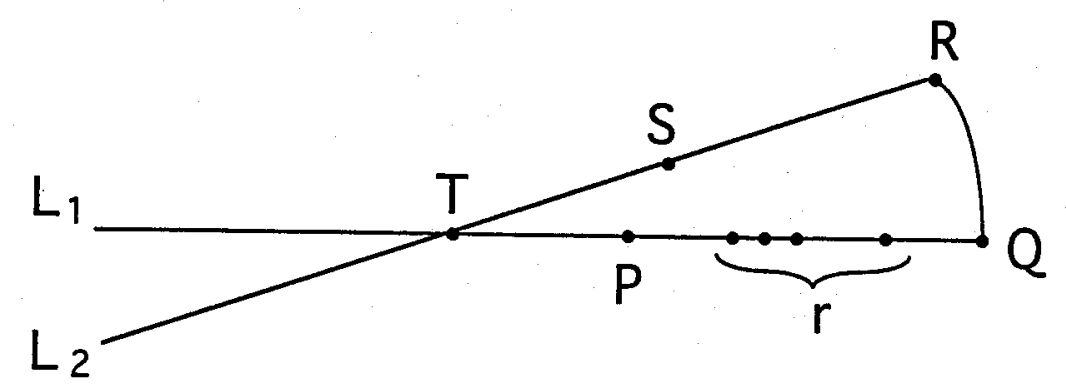

